\title{
Anthropmetric Profiles of Elite Triathletes
}

\author{
T.R. Ackland, B.A. Blanksby G. Landers \& D. Smith* \\ Department of Human Movement, The University of Western Australia \& Triathlon \\ Australia, Australian Sports Commission*
}

Ackland T.R., Blanksby B.A., Landers G. \& Smith D. (1997) Anthropmetric Profiles of Elite Triathletes. Journal of Science and Medicine in Sport 1(1): 51-56

\section{Introduction}

The sport of triathlon is relatively new, having originated only 20 years ago, but has become one of the world's fastest growing participation sports. It caters for all ages from 10 years to veterans and will be conducted at the Olympics for the first time in Sydney 2000. Triathlon consists of the three disiplines of swimming, cycling and running performed continuously in the above sequence. The literature reveals that only a few studies have been carried out on triathletes and these are mostly on ultra-endurance or beginner triathletes. Coaches are questioning which body types, proportions or shapes are best suited for each of the three components of the triathlon. There is no evidence as to whether the champion triathlete is equally an "all rounder" in the three sub-sections or whether an exceptional performance in one discipline can compensate for only average ability in the other two disciplines. Therefore, the identification of a particular body type which might provide an advantage for triathletes could enhance the coaching process of selecting athletes having the preferred morphology and proportionality.

No kinanthropometric data have been reported for triathletes at the World Championship level, but two studies have been made on small samples at the Hawaiian Ironman, an ultra endurance event lasting in excess of 8 hours (Holly et al., 1986; OToole et al., 1987). Other studies have focused on women competitors (Zingraf et al., 1986; Leake and Carter, 1991; De Vito et al.,1995) but these did not include elite athletes and the number of variables reported was limited. Leake and Carter (1991) reported that the mean somatotype of competitive female triathletes $(n=16)$ was $3.1-4.3-2.6$, or a balanced mesomorphy. According to the authors, triathletes were less ectomorphic and more mesomorphic than middle distance runners, and recorded greater skinfold thickness at all of the six sites tested. The triathletes were also found to be heavier than the runners but lighter than swimmers, thereby suggesting a crosstraining effect. There was no significant difference in somatotype or skinfold patterning of the swimmers and triathletes.

The aim of this project was to profile elite senior and junior triathletes competing in the 1997 World Triathlon Championships held in Perth, Western Australia. This event was held over the "classic" distances of a $1500 \mathrm{~m}$ swim, 
$40 \mathrm{~km}$ cycle and $10 \mathrm{~km}$ run. The overall project forms part of a comparison of triathletes with their counterparts in the discrete disciplines of swimming, cycling and running in terms of body type, composition and proportionality. However, this short communication presents the descriptive statistics of triathletes which may be used for athlete profiling.

\section{Methods}

Competitors from 11 nations competing in the 1997 World Triathlon Championships were measured on a battery of 28 anthropometric dimensions. A sample size of 87 acted as subjects. In all, data were recorded from 19 male and 19 female senior elite, as well as 29 male and 20 female junior elite competitors.

Testing was carried out at the Department of Human Movement laboratories at The University of Western Australia when the athletes were able to attend. Otherwise, a mobile laboratory was despatched to accommodation and training venues for each of the teams so that triathletes could be measured in a single session during a rest-time within their training schedule. Most athletes were measured in the seven day period prior to the championships with another four measured after the completion of the event. As a result, their level of motivation was high and good cooperation with team management ensured a smooth data gathering process.

After landmarking by a criterion anthropometrist, each triathlete was directed to one of five stations for the measurement of eight skinfolds, seven direct lengths, seven segment girths, five segment breadths plus body mass. All variables were measured on the right side of the body and the median score for skinfolds (measured in triplicate), or mean score for other variables (duplicate measures) were recorded. The standard procedures for each measurement, as supported by the International Society for the Advancement of Kinanthropometry (ISAK) and reported in Bloomfield et al. (1994) and Norton and Olds (1996) were followed at all times. All measurements were made by trained anthropometrists whose technical errors of measurement were were within the target values recommended by Carter and Ackland (1994) for the KASP study.

Finally, as a service to the participating subjects and their team management, personal profiles were produced and supplied to each individual. These profiles provided individual data which can then be compared with average values for the sample sub-sections included in this brief paper.

\section{Results \& Discussion}

Mean values for a selection of absolute size variables are presented in Table 1 , with triathletes separated by competition age groups and gender. It should be noted that no measures of maturity were recorded and the data are presented in groups who compete against each other. The elite juniors ranged from 16-19 years and could include several athletes who had not reached full maturity. Hence, the percentile scores should be read with that in mind because it could tend to skew some of the measures if used for talent identification or normative data. The availability of normative data with which one may create a physical profile of the athlete can be of substantial utility for the coach and sports scientist. This information is presented in Tables 2 and 3 as percentile scores.

Profiling allows for the assessment of the athlete on a regular basis, usually corresponding with a particular training phase which assists in monitoring 
Short Communication - Anthropometric Profiles of Elite Triathletes

\begin{tabular}{|c|c|c|c|c|c|}
\hline \multirow[b]{2}{*}{ MEASURE* } & & \multicolumn{2}{|c|}{ FEMALES } & \multicolumn{2}{|c|}{ MALES } \\
\hline & & $\begin{array}{c}\text { Elite } \\
(n=19)\end{array}$ & $\begin{array}{c}\text { Junlor } \\
(\mathbf{n}=20)\end{array}$ & $\begin{array}{c}\text { Ellte } \\
(n=19)\end{array}$ & $\begin{array}{c}\text { Junior } \\
(\mathbf{n}=29)\end{array}$ \\
\hline Age & $\begin{array}{l}\bar{X} \\
\text { SD }\end{array}$ & $\begin{array}{r}28.8 \\
3.0\end{array}$ & $\begin{array}{r}18.4 \\
1.5\end{array}$ & $\begin{array}{r}26.3 \\
4.4\end{array}$ & $\begin{array}{r}18.7 \\
1.1\end{array}$ \\
\hline Stature & $\begin{array}{l}\bar{X} \\
\text { SD }\end{array}$ & $\begin{array}{r}168.3 \\
4.4\end{array}$ & $\begin{array}{r}164.9 \\
7.2\end{array}$ & $\begin{array}{r}180.1 \\
5.9\end{array}$ & $\begin{array}{r}175.7 \\
5.7\end{array}$ \\
\hline Body Mass & $\begin{array}{l}\bar{X} \\
\text { SD }\end{array}$ & $\begin{array}{r}59.3 \\
4.7\end{array}$ & $\begin{array}{r}56.7 \\
5.4\end{array}$ & $\begin{array}{r}72.6 \\
6.0\end{array}$ & $\begin{array}{r}67.2 \\
6.3\end{array}$ \\
\hline Sum 8 Skinfolds & $\begin{array}{l}\bar{X} \\
S D\end{array}$ & $\begin{array}{l}62.8 \\
13.4\end{array}$ & $\begin{array}{l}73.1 \\
17.2\end{array}$ & $\begin{array}{l}48.3 \\
10.2\end{array}$ & $\begin{array}{l}51.8 \\
14.8\end{array}$ \\
\hline Arm Span & $\begin{array}{l}\bar{X} \\
\text { SD }\end{array}$ & $\begin{array}{r}170.6 \\
4.3\end{array}$ & $\begin{array}{r}166.8 \\
7.4\end{array}$ & $\begin{array}{r}186.6 \\
6.4\end{array}$ & $\begin{array}{r}180.9 \\
8.4\end{array}$ \\
\hline Arm Length & $\begin{array}{l}\bar{X} \\
S D\end{array}$ & $\begin{array}{r}31.8 \\
1.2\end{array}$ & $\begin{array}{r}31.1 \\
1.6\end{array}$ & $\begin{array}{r}34.4 \\
1.4\end{array}$ & $\begin{array}{r}33.2 \\
1.6\end{array}$ \\
\hline Forearm Length & $\begin{array}{l}\bar{X} \\
\text { SD }\end{array}$ & $\begin{array}{r}24.2 \\
1.2\end{array}$ & $\begin{array}{r}23.9 \\
1.5\end{array}$ & $\begin{array}{r}26.7 \\
1.1\end{array}$ & $\begin{array}{r}26.4 \\
1.9\end{array}$ \\
\hline Hand Length & $\begin{array}{l}\bar{X} \\
S D\end{array}$ & $\begin{array}{r}18.4 \\
0.9\end{array}$ & $\begin{array}{r}17.4 \\
0.8\end{array}$ & $\begin{array}{r}19.4 \\
0.6\end{array}$ & $\begin{array}{r}19.2 \\
0.9\end{array}$ \\
\hline Thigh Length ${ }^{\dagger}$ & $\begin{array}{l}\bar{X} \\
S D\end{array}$ & $\begin{array}{r}43.6 \\
2.6\end{array}$ & $\begin{array}{r}43.7 \\
2.9\end{array}$ & $\begin{array}{r}46.8 \\
2.0\end{array}$ & $\begin{array}{r}44.4 \\
2.1\end{array}$ \\
\hline Leg Length ${ }^{\dagger}$ & $\begin{array}{l}\bar{X} \\
S D\end{array}$ & $\begin{array}{r}43.8 \\
1.9\end{array}$ & $\begin{array}{r}43.0 \\
2.6\end{array}$ & $\begin{array}{r}47.8 \\
2.1\end{array}$ & $\begin{array}{r}46.3 \\
2.1\end{array}$ \\
\hline Flexed Arm Girth & $\begin{array}{l}\bar{X} \\
\text { SD }\end{array}$ & $\begin{array}{r}28.2 \\
1.3\end{array}$ & $\begin{array}{r}27.7 \\
1.5\end{array}$ & $\begin{array}{r}31.2 \\
2.7\end{array}$ & $\begin{array}{r}31.1 \\
1.9\end{array}$ \\
\hline Forearm Girth & $\begin{array}{l}\bar{X} \\
\text { SD }\end{array}$ & $\begin{array}{r}23.8 \\
1.0\end{array}$ & $\begin{array}{r}23.6 \\
1.0\end{array}$ & $\begin{array}{r}26.8 \\
1.0\end{array}$ & $\begin{array}{r}26.2 \\
1.1\end{array}$ \\
\hline Chest Girth & $\begin{array}{l}\bar{X} \\
S D\end{array}$ & $\begin{array}{r}88.0 \\
2.7\end{array}$ & $\begin{array}{r}86.4 \\
2.9\end{array}$ & $\begin{array}{r}99.6 \\
3.6\end{array}$ & $\begin{array}{r}94.6 \\
4.6\end{array}$ \\
\hline Waist Girth & $\begin{array}{l}\bar{X} \\
S D\end{array}$ & $\begin{array}{r}67.9 \\
3.5\end{array}$ & $\begin{array}{r}67.6 \\
2.7\end{array}$ & $\begin{array}{r}77.8 \\
2.8\end{array}$ & $\begin{array}{r}74.9 \\
3.8\end{array}$ \\
\hline Hip Girth & $\begin{array}{l}\bar{X} \\
S D\end{array}$ & $\begin{array}{r}90.8 \\
3.3\end{array}$ & $\begin{array}{r}89.9 \\
3.0\end{array}$ & $\begin{array}{r}93.0 \\
3.0\end{array}$ & $\begin{array}{r}90.9 \\
3.6\end{array}$ \\
\hline Thigh Girth & $\begin{array}{l}\bar{X} \\
\text { SD }\end{array}$ & $\begin{array}{r}54.0 \\
3.6\end{array}$ & $\begin{array}{r}53.8 \\
2.1\end{array}$ & $\begin{array}{r}55.4 \\
3.3\end{array}$ & $\begin{array}{r}53.3 \\
2.8\end{array}$ \\
\hline Calf Girth & $\begin{array}{l}\bar{X} \\
S D\end{array}$ & $\begin{array}{r}35.4 \\
1.8\end{array}$ & $\begin{array}{r}33.6 \\
3.2\end{array}$ & $\begin{array}{r}36.9 \\
1.6\end{array}$ & $\begin{array}{r}36.0 \\
2.1\end{array}$ \\
\hline Biacromial Breadth & $\begin{array}{l}\bar{X} \\
S D\end{array}$ & $\begin{array}{r}36.5 \\
2.3\end{array}$ & $\begin{array}{r}36.9 \\
1.2\end{array}$ & $\begin{array}{r}41.5 \\
1.8\end{array}$ & $\begin{array}{r}40.5 \\
1.3\end{array}$ \\
\hline Transverse Chest Breadth & $\begin{array}{l}\bar{X} \\
S D\end{array}$ & $\begin{array}{r}26.9 \\
0.9\end{array}$ & $\begin{array}{r}26.6 \\
1.1\end{array}$ & $\begin{array}{r}30.2 \\
1.2\end{array}$ & $\begin{array}{r}28.7 \\
1.0\end{array}$ \\
\hline Biiliocristal Breadth & $\begin{array}{l}\bar{X} \\
S D\end{array}$ & $\begin{array}{r}27.6 \\
1.1\end{array}$ & $\begin{array}{r}26.7 \\
1.2\end{array}$ & $\begin{array}{r}28.3 \\
1.2\end{array}$ & $\begin{array}{r}27.2 \\
1.4\end{array}$ \\
\hline Humerus Breadth & $\begin{array}{l}\bar{X} \\
S D\end{array}$ & $\begin{array}{l}6.3 \\
0.3\end{array}$ & $\begin{array}{l}6.2 \\
0.3\end{array}$ & $\begin{array}{l}7.2 \\
0.3\end{array}$ & $\begin{array}{l}6.9 \\
0.4\end{array}$ \\
\hline Femur Breadth & $\begin{array}{l}\bar{X} \\
S D\end{array}$ & $\begin{array}{l}9.0 \\
0.8\end{array}$ & $\begin{array}{l}9.0 \\
0.3\end{array}$ & $\begin{array}{l}9.8 \\
0.4\end{array}$ & $\begin{array}{l}9.8 \\
0.4\end{array}$ \\
\hline
\end{tabular}

Table 1: $\quad$ Absolute Size Scores for Elite and Junior Triathletes 


\begin{tabular}{|lrrrrrrrr|}
\hline & \multicolumn{7}{c}{ PERCENTILES } \\
MEASURE & $\mathbf{5}$ & \multicolumn{1}{c}{$\mathbf{1 0}$} & $\mathbf{3 0}$ & \multicolumn{1}{c|}{$\mathbf{5 0}$} & \multicolumn{1}{c|}{$\mathbf{0}$} & $\mathbf{9 0}$ & $\mathbf{9 5}$ \\
\hline Stature (cm) & 154.2 & 158.2 & 164.2 & 168.2 & 169.8 & 174.8 & 176.8 \\
Body Mass (kg) & 49.8 & 51.7 & 55.2 & 56.9 & 62.4 & 65.0 & 65.8 \\
Sum 8 Skinfolds (mm) & 40.3 & 46.4 & 59.5 & 66.1 & 75.2 & 95.8 & 98.4 \\
Arm Span (cm) & 159.2 & 163.0 & 166.0 & 169.2 & 172.4 & 178.0 & 180.9 \\
Arm Length (cm) & 29.0 & 29.2 & 31.0 & 31.4 & 32.5 & 33.5 & 34.4 \\
Forearm Length (cm) & 21.9 & 22.5 & 23.5 & 24.0 & 24.8 & 26.0 & 26.1 \\
Hand Length (cm) & 16.2 & 16.6 & 17.5 & 18.0 & 18.4 & 19.4 & 19.8 \\
Thigh Length (cm) & 38.6 & 40.7 & 42.5 & 43.7 & 45.0 & 47.7 & 48.7 \\
Leg Length (cm) & 38.4 & 40.9 & 42.6 & 43.4 & 45.0 & 46.4 & 48.3 \\
Flexed Arm Girth (cm) & 25.8 & 26.2 & 27.2 & 27.9 & 28.8 & 30.1 & 30.8 \\
Forearm Girth (cm) & 21.6 & 22.6 & 23.2 & 23.6 & 24.4 & 25.1 & 25.2 \\
Chest Girth (cm) & 83.1 & 83.6 & 85.1 & 87.0 & 89.5 & 91.6 & 92.3 \\
Waist Girth (cm) & 62.8 & 63.6 & 65.3 & 68.4 & 69.8 & 72.6 & 73.3 \\
Hip Girth (cm) & 85.5 & 86.5 & 88.6 & 90.0 & 92.6 & 95.2 & 95.8 \\
Thigh Girth (cm) & 51.0 & 51.4 & 52.0 & 54.0 & 55.6 & 57.3 & 58.5 \\
Calf Girth (cm) & 31.0 & 31.6 & 34.1 & 34.8 & 35.8 & 37.7 & 38.0 \\
Biacromial Breadth (cm) & 33.9 & 35.6 & 36.1 & 37.0 & 37.7 & 38.6 & 38.8 \\
Chest Breadth (cm) & 25.2 & 25.5 & 26.1 & 26.6 & 27.4 & 28.4 & 28.6 \\
Biiliocristal Breadth (cm) & 24.9 & 25.5 & 26.8 & 27.2 & 27.9 & 29.1 & 29.5 \\
Humerus Breadth (cm) & 5.6 & 5.9 & 6.0 & 6.2 & 6.3 & 6.6 & 6.8 \\
Femur Breadth (cm) & 8.4 & 8.6 & 8.9 & 9.0 & 9.2 & 9.5 & 9.8 \\
\hline
\end{tabular}

Table 2: $\quad$ Percentile scores for combined elite and junior female triathletes $(n=39)$.

\begin{tabular}{|c|c|c|c|c|c|c|c|}
\hline \multirow[b]{2}{*}{ MEASURE } & \multicolumn{7}{|c|}{ PERCENTILES } \\
\hline & 5 & 10 & 30 & 50 & 70 & 90 & 95 \\
\hline Stature $(\mathrm{cm})$ & 168.8 & 170.0 & 174.5 & 178.4 & 180.0 & 186.8 & 190.8 \\
\hline Body Mass (kg) & 59.1 & 60.6 & 66.5 & 68.9 & 74.4 & 80.2 & 81.6 \\
\hline Sum 8 Skinfolds (mm) & 36.8 & 38.2 & 43.5 & 47.6 & 53.0 & 67.2 & 85.9 \\
\hline Arm Span $(\mathrm{cm})$ & 168.8 & 172.8 & 178.9 & 183.1 & 190.0 & 196.2 & 197.0 \\
\hline Arm Length $(\mathrm{cm})$ & 30.9 & 31.3 & 33.0 & 33.8 & 34.8 & 36.3 & 36.8 \\
\hline Forearm Length $(\mathrm{cm})$ & 24.6 & 24.7 & 25.8 & 26.4 & 27.1 & 28.4 & 28.6 \\
\hline Hand Length $(\mathrm{cm})$ & 18.0 & 18.1 & 18.8 & 19.4 & 19.7 & 20.4 & 20.7 \\
\hline Thigh Length $(\mathrm{cm})$ & 42.2 & 42.7 & 43.8 & 45.3 & 47.1 & 49.3 & 50.4 \\
\hline Leg Length $(\mathrm{cm})$ & 42.3 & 43.9 & 45.6 & 47.0 & 48.6 & 50.4 & 51.4 \\
\hline Flexed Arm Girth (cm) & 27.8 & 28.4 & 30.5 & 31.6 & 32.5 & 33.9 & 34.8 \\
\hline Forearm Girth (cm) & 24.1 & 24.8 & 25.8 & 26.4 & 27.1 & 28.0 & 28.3 \\
\hline Chest Girth (cm) & 86.8 & 90.4 & 94.2 & 96.8 & 99.6 & 103.2 & 105.2 \\
\hline Waist Girth (cm) & 70.3 & 71.2 & 74.2 & 76.4 & 78.3 & 80.2 & 81.9 \\
\hline Hip Girth (cm) & 86.6 & 87.0 & 89.6 & 92.2 & 94.6 & 97.2 & 98.2 \\
\hline Thigh Girth $(\mathrm{cm})$ & 49.8 & 50.6 & 52.4 & 54.0 & 55.8 & 58.9 & 60.1 \\
\hline Calf Girth $(\mathrm{cm})$ & 33.2 & 33.6 & 35.5 & 36.5 & 37.5 & 39.4 & 39.7 \\
\hline Biacromial Breadth $(\mathrm{cm})$ & 38.5 & 38.7 & 39.9 & 41.0 & 41.6 & 43.5 & 44.0 \\
\hline Chest Breadth $(\mathrm{cm})$ & 27.0 & 27.6 & 28.8 & 29.4 & 30.0 & 31.5 & 32.0 \\
\hline Billiocristal Breadth (cm) & 25.8 & 26.0 & 26.7 & 27.7 & 28.6 & 30.1 & 30.4 \\
\hline Humerus Breadth $(\mathrm{cm})$ & 6.4 & 6.5 & 6.8 & 7.1 & 7.2 & 7.6 & 7.8 \\
\hline Femur Breadth $(\mathrm{cm})$ & 9.1 & 9.2 & 9.6 & 9.8 & 10.1 & 10.4 & 10.8 \\
\hline
\end{tabular}

Table 3: Percentile scores for combined elite and junior male triathletes $(n=48)$. 
status, identifying areas that may need corrective or remedial work, and developing and planning training programs which are specifically for the individual. Whilst many of the variables presented in this paper cannot be altered with training, those which relate to adiposity, body mass, and segment girths and breadths may be modified over time. These are the variables (together with other physiological parameters) which are of most utility in the preparation of elite performers.

Parameters such as the segment lengths and breadths, which are less easily modified, tend to be more useful for comparing younger athletes from development squads and assist coaches to develop appropriate techniques which are tailored to suit individual proportions. Since human proportions appear to remain stable from late adolescence, and even from early adolescence for the upper limb variables (Ackland and Bloomfield, 1996), these structural variables may be useful for talent identification and development purposes.

\section{References}

Ackland, T.R. and Bloomfield, J. (1996) Stability of human proportions through adolescent growth. The Australian Journal of Science and Medicine in SporT, 28(2): 57-60.

Bloomfield, J., Ackland, T.R. and Elliott, B.C. (1994) Applied Anatomy and Biomechanics in Sport, Blackwell Scientific Publications, Melbourne.

Carter, J.E.L. and Ackland, T.R. (1994) Kinanthropometry in Aquatic Sports. Human Kinetics Publishers, Champaign, Illinois.

De Vito, G., Bernardi, M. and Sproiero, E. (1995) Decrease in endurance performance during Olympic triathlon. International Journal of Sports Medicine, 16(1): 24-28.

Holly, R.G., Barnard, R.J., Rosenthal, M., Applegate, E. and Pritikin, N. (1986) Triathlete characterisation and response to prolonged strenuous competition. Medicine and Science in Sports and Exercise, 18(1): 123-127.

Leake, C.N. and Carter, J.E.L. (1991) Comparison of body composition and somatotypes of trained female triathletes. Journal of Sports Sciences, 9: 125-135.

Norton, K. and Olds, T. (1996) Anthropometrica: A Textbook of Body Measurement for Sports and Health Courses, University of new South Wales Press, Sydney.

OToole, M.L., Douglas, E., Hiller, B., Crosby, L.O. and Douglas, P.S. (1987) The ultra endurance triathlete: A physiological profile. Medicine and Science in Sports and Exercise, 19(1): 4550.

Zingraf, S., Jones, C. and Warren B. (1986) An emperical investigation of triathlon performance. Journal of Sports Medicine and Physical Fitness, 26: 350-356. 